DOI 10.35381/cm.v5i9.146

\title{
Perspectiva neurológica de la lectoescritura durante el aprendizaje escolar
}

\author{
Neurological perspective of literacy during school learning
}

\author{
Deysi Rosario Basantes Moscoso \\ dbasantes@unach.edu.ec \\ Universidad Nacional del Chimborazo \\ Ecuador \\ https://orcid.org/0000-0001-8742-8226 \\ Guillermo Gonzalo Gualpa Jaramillo \\ ggualpa@unach.edu.ec \\ Universidad Nacional de Chimborazo \\ Ecuador \\ https://orcid.org/0000-0003-1752-6642 \\ Guillermo Euclides Galecio Samaniego \\ guillermo.galecios@ug.edu.ec \\ Universidad Laica Vicente Rocafuerte \\ Ecuador \\ https://orcid.org/0000-0002-9638-3311
}

Recibido: 1 de mayo de 2019

Aprobado: 14 de junio de 2019

\begin{abstract}
RESUMEN
Una mala valoración del test de APGAR al momento del nacimiento, no permite predecir tempranamente las dificultades de aprendizaje relacionadas al proceso lectoescritor, cuadros de hipoxia y asfixia, sumado a ello la falta de estimulación durante la vida intrauterina, partos y cesáreas mal practicados, consumo de tabaco y alcohol durante la gestación, el ambiente socio-cultural y la escuela; son factores que influyen en el aprendizaje de la lectura. Es necesario que el grupo obstetra derive a centros de estimulación temprana para la ejecución de terapias que ayuden al niño a superar dificultades de aprendizaje, problemas motores, sensoriales y de adaptación. Aunado a un buen manejo humano - pedagógico en la escuela, lo cual permitirá contribuir en la integración del estudiante, así como de motivarse al aprendizaje, adquisición de
\end{abstract}


hábito de lectura como medio para aprender para la vida, ejecutándose una educación inclusiva de calidad.

Descriptores: Deserción escolar; Trastornos del habla; Fonética; Educación; Atención.

\begin{abstract}
A poor assessment of the APGAR test at birth does not allow early prediction of learning difficulties related to the reading-writing process, hypoxia and asphyxiation, added to this the lack of stimulation during intrauterine life, births and caesarean sections poorly practiced, tobacco and alcohol consumption during pregnancy, the socio-cultural environment and the school; they are factors that influence the learning of reading. It is necessary for the obstetrician group to refer to early stimulation centers for the execution of therapies that help the child overcome learning difficulties, motor, sensory and adaptation problems. Together with a good human - pedagogical management in the school, which will contribute to the integration of the student, as well as to motivate the learning, acquisition of reading habit as a means to learn for life, executing a quality inclusive education.
\end{abstract}

Descriptors: Dropping out; Speech disorders; Phonetics; Education; Attention.

\title{
INTRODUCCIÓN
}

El valor que tiene la adquisición del proceso lector en los años de escolaridad es decisivo, porque de este dependerá el éxito y/o fracaso de los distintos procesos académicos que se dan dentro y fuera del aula. El lenguaje es el instrumento primordial para los aprendizajes y la interacción con otros. A través del mismo se desarrolla el conocimiento, siendo uno de los requisitos básicos para obtener el éxito en la trayectoria escolar. Así lo manifiesta Piaget (1987):

Durante los tres primeros meses de vida las rutinas ligadas a las necesidades del bebé y el adulto comparten significados que permiten regular conjuntamente algunas de sus conductas. En esta relación específica que hay entre el cuidador (madre u otro miembro) y el niño, la madre empieza a enseñar inconscientemente el lenguaje. Esto ocurre comenzando en el nivel más fundamental y haciéndose gradualmente más complejo a medida que el niño crece. El habla se caracteriza por ser simple, frases cortas, con un alto grado de redundancia; las palabras utilizadas son aquellas que están presentes en el entorno del niño, hay preguntas y explicaciones sobre lo que la madre va diciendo o lo que el niño hace 
Un niño estimulado desde los primeros meses de vida, deseado desde el momento de su concepción, involucrado en aprendizajes lectores, con padres preocupados por el futuro cultural y académico, a diferencia de un niño que crece en el abandono, desprotegido, al margen del descuido, recibiendo información en aulas frías, sin los instrumentos y herramientas necesarias para construir conocimientos y niveles intelectuales que lo involucren en el fascinante mundo lector, posee mayor posibilidad de ser un exitoso lectoescritor, competencia transversal que le permite desarrollar un aprendizaje critico - reflexivo en posibilidad de transcender la escuela efectivamente, etapa que le permitirá proseguir con sus estudios con habilidades posibilitadoras de triunfar en el resto de la educación formal.

\title{
Score de APGAR
}

Al momento del nacimiento se le practica una prueba al infante con la finalidad de medir su condición o estado general, Luna (2005) describe que:

\begin{abstract}
En 1952, Virginia Apgar, propuso el score de APGAR como un medio para evaluar la condición física de los neonatos inmediatamente después de nacidos. Por ser de fácil realización y un excelente indicador de la condición del neonato, rápidamente se difundió en todo el mundo e incluso actualmente se continúa utilizando. Se realiza al minuto y a los 5 minutos. De los 2 scores, el de los 5 minutos es considerado como el mejor predictor de sobrevida en la infancia (p. 1).
\end{abstract}

Esta prueba permite a los médicos evaluar la respuesta general del infante ante el contexto al cual se enfrenta como recién nacido, prediciendo si necesita atención de urgencia o por el contario se procede a un control estandarizado. Por otro lado, Myklebus creador de la teoría de la integración perceptiva, intervención de las neurofunciones: visión, audición y motora logran la coordinación ideográfica, visográfica y audiográfica. Pulsando las tres modalidades logramos la percepción de modalidad cruzada, la sobrecarga de una de las áreas produce errores específicos de aprendizaje.

La evaluación cerebral en el infante recién nacido es de suma importancia para conocer la funcionalidad de este importante órgano con la finalidad de prever el 


\section{CIENCIAMATRIA}

Revista Interdisciplinaria de Humanidades, Educación, Ciencia y Tecnología

Año V. Vol. V. Nº. Julio - Diciembre 2019

Hecho el depósito de ley: pp201602FA4721

ISSN-L: 2542-3029; ISSN: 2610-802X

Universidad Nacional Experimental Francisco de Miranda (UNEFM). Santa Ana de Coro. Venezuela

Deysi Rosario Basantes Moscoso; Guillermo Gonzalo Gualpa Jaramillo; Guillermo Euclides Galecio Samaniego

tratamiento de posibles trastornos que puedan afectar el desenvolvimiento del infante en su progresiva etapa de socialización, Luria (1973), destaca que:

Un trastorno específico de aprendizaje es un DCM (Daño Cerebral Mínimo), producida por la falta de irrigación del primer bloque atención y memoria localizadas en la primera y segunda circunvolución frontal y las áreas de asociación frontal y parietal. El segundo bloque de la percepción localizado en la primera circunvolución parietal; y el tercer bloque constituyen las motivaciones.

La integridad de las estructuras cerebrales relacionadas con las funciones cognitivas determina la capacidad de aprendizaje, la amígdala relacionada con el proceso emocional, la corteza prefrontal dorso lateral en la toma de decisiones y la corteza frontal en la cual las conexiones neuronales se siguen desarrollando hasta las edades de 11 años en el ser humano, con aumento de la mielinización axonal, produce un incremento en la velocidad de conducción nerviosa y eficiencia neuronal con especificidad de acciones principalmente de tipo ejecutivas sitio especifico de almacenamiento de la memoria inmediata, los recuerdos y las actividades que se utilizaran en etapas posteriores.

Sin embargo se almacenaran en la amígdala principalmente en el hasta de Amon, del lóbulo temporal mismo que además produce altos niveles de dopamina, hormona relacionada con la felicidad, esta capacidad tiene un complejo sistema de conexiones neuronales que van desde su almacenamiento inicial incipiente e involucra a estructuras denominadas sistema límbico que incluye, fornix, hipocampo, tálamo, hipotálamo, cuerpo geniculado externo, asta de Amon y amígdala cerebral del lóbulo temporal, en la cual se almacenan principalmente la memoria de eventos pasados.

En procesos de falta de madurez o falta de migración neuronal adecuada estas funciones en los órganos referidos producen dificultades cognitivas, sobre todo al momento de nacer por lo que requiere la adecuada atención del parto que evita lesiones corticales y subcorticales por asfixia perinatal. Las estructuras corticales cerebrales relacionadas con la escritura también son sistemáticas organizadas y secuenciales en las que intervienen áreas tanto corticales como subcorticales de núcleos altamente especializados de células nerviosas integradas en el complejo 
sistema de vías tanto piramidal, extra piramidal y conexiones cortas entre núcleos: caudado, globo pálido, Putámen y cerebelo.

La recepción de la información visual auditiva, kinestésica llegan por vías aferentes a la corteza motora primaria, en la circunvolución frontal ascendente áreas de Brodman 4 es analizada en la corteza motora secundaria y luego procesada en la corteza motora suplementaria, áreas de Brodman 6 y 1, 2 y 3 en el lóbulo temporal, esta información receptada analizada y procesada son ejecutadas en acciones motrices finas o gruesas, a través de Putámen, caudado y globo pálido denominados núcleos basales, en coordinación con el cerebelo, e intervienen una serie de mediadores, tanto en la vía piramidal voluntaria como en la vía Extra piramidal involuntaria, mediadores tipo serotoninérgicos y dopaminérgicos.

La estimulación temprana, y el gusto por la lectura, constituyen factores decisivos en el futuro lector, he ahí la responsabilidad de los padres, la escuela y el medio ambiente por formar niños lectores, con prácticas de comprensión, imaginación, análisis, pero sobre todo de disfrute e involucramiento en el hecho lector y comunicacional, sin embargo, el Instituto Nacional de Estadística y Censos (INEC, 2012), destaca que "el $26,5 \%$ de los ecuatorianos no dedica tiempo para leer, de ellos el $56,8 \%$ no lo hace por falta de interés y el $31,7 \%$ por falta de tiempo", esto evidencia una falta de cultura hacia la lectura, aunado a posibles trastornos neurológicos o físicos que impidan al estudiante leer efectivamente, se constituyen en indicadores que el sistema educativo debe tener en consideración para adoptar medidas que permitan incrementar el hábito por la lectura.

Bajo estas consideraciones un niño/a debe aprender a leer desde el momento que ingresa a la escuela, "situación retrograda", un niño desde sus primeros meses de vida intrauterina, percibe estímulos, positivos y negativos, he ahí la madre la primera responsable en estimular el gusto por la lectura. En prosecución de lo planteado, Hernández Velázquez, Landrove Borjas \& Andrés Matos (2014), destacan que:

Estudios realizados sobre desórdenes psicomotores en niños con antecedentes de hipoxia perinatal al nacer sostienen que: los neonatos por medio de cesárea que han sufrido asfixia durante el parto, presentaron alteración en el desarrollo psicomotor, el cual 55,6 \% de los pacientes 


\section{CIENCIAMATRIA}

Revista Interdisciplinaria de Humanidades, Educación, Ciencia y Tecnología

Año V. Vol. V. Nº. Julio - Diciembre 2019

Hecho el depósito de ley: pp201602FA4721

ISSN-L: 2542-3029; ISSN: 2610-802X

Universidad Nacional Experimental Francisco de Miranda (UNEFM). Santa Ana de Coro. Venezuela

Deysi Rosario Basantes Moscoso; Guillermo Gonzalo Gualpa Jaramillo; Guillermo Euclides Galecio Samaniego

evidenciaron algún tipo de alteración, distribuidas de la siguiente manera: $16,7 \%$ presentaban retardo psicomotor ligero, el $27,8 \%$, retardo moderado y el $11,1 \%$ severo, observándose un alto índice de un retraso moderado en la mayoría de los niños además que estas alteraciones predominaron en varones. Según Little a mediados del siglo XIX, los autores que han señalado la asfixia como la causa que, al actuar en el cerebro del feto y el recién nacido, puede originar lesiones orgánicas que condicionarán posteriormente la aparición de retraso psicomotor.

Estas lesiones pueden originar lesiones psicomotoras que afectan no solo el sano desarrollo bilógico del infante, sino, que genera amenazas sobre la posibilidad de adquirir competencias de lecto escritura pertinentes para configurar un accionar pedagógico favorable en comparación con niños que no han sufrido este tipo de afecciones. Por otro lado, Podestá L, et. al. (2013).

Afirman que en mujeres en las que se les practicó cesárea puede producirse depresión post-parto y está ocasionará en el niño un leve retraso motor ya que la falta de apego o patrones de interacción negativos madrehijo, durante la etapa temprana de formación de vínculos, impactan el desarrollo futuro del niño, aun cuando la depresión de la madre haya finalizado, esto se da porque se separa al infante durante una hora después del parto, es aquí donde se puede tener una relación madre e hijo. Además, a los 18 meses estos niños presentan un peor desempeño en la escala de evaluación de desarrollo psicomotor de Bayley

La repuesta afectiva de la madre puede influir positiva o negativamente en la formación de un lecto escritor con competencias favorables para la generación de un estudiante en capacidad de desarrollar hábitos favorables de lectura con la finalidad de promover una educación critica - reflexiva desde la lectura. Las dificultades de aprendizaje tratadas desde el punto de vista neuropsicológico permiten a muchos profesionales de la salud y educación entender y tratar adecuadamente los efectos que se producen en el estudiante durante su etapa de escolaridad. El aporte científico, médico, psicológico, pediátrico, educativo, cognitivo que la neurociencia brinda en la actualidad al sector de la salud y de la educación, es impresionante, sin embargo se carece de información, y de conocimiento sobre la relación cerebro-cognición, dependiendo del tipo de dificultad de aprendizaje al que el pre-escolar se ve sujeto.

Al tener un conocimiento pleno de la importancia del estudio y aplicación de la neuropsicología, pero sobre todo la aplicación responsable, correcta y a tiempo de 
una valoración de APGAR (apariencia, pulso, gesticulación, actividad y respiración),después del minuto a cinco minutos del nacimiento, se garantiza al neonato un futuro saludable y evita una serie de partos mal atendidos, existe menos riesgo de mortalidad infantil por mala práctica médica, se ha podido prevenir niños con riesgos biológicos, anulando la posibilidad de presentar déficits neurocognitivos, además ha permitido rehabilitar a niños con daños cerebrales, permitiéndoles tener una mejor calidad de vida y por supuesto mejorar las relaciones emocionales y sociales de la familia.

\section{Dificultades específicas de aprendizaje}

En el proceso educativo un niño normal presenta dificultades de orden afectivo, social, ambiental por tal motivo no les permite un desarrollo o desenvolvimiento normal en el medio. No deben ser considerados como diferentes sino como entes con diferentes discapacidades, sin embargo, a juicio de Scrich Vázquez, et. al. (2017), destacan que

El sistema educativo ecuatoriano no está preparado para diagnosticar ni tratar a alumnos con trastornos psíquicos como la dislexia, la disgrafia y la discalculia. La desmotivación por el estudio, la baja autoestima y la deserción escolar en este tipo de alumno es una de las consecuencias más notables en todos los niveles de enseñanza, por lo que merece una intervención científica inmediata (p. 770).

Lo planteado, indica la urgente necesidad de asumir estrategias que permitan estructurar acciones en conformidad de articular en las instituciones educativas la posibilidad de afrontar desde una perspectiva multidimensional, la problemática planteada, siendo necesaria el abordaje de la neurología en el aprendizaje de los estudiantes, por cuanto esto permite promover una psicopedagogía que atienda en pertinencia a los retos educativos de inclusión para el buen vivir, esto obliga a los docentes a conocer y formarse en el campo neurológico con la finalidad de conocer las diversas discapacidades que puede padecer el estudiante, más aun, como abordarlas, en este sentido, lanni Gómez (2018), complementa al indicar que "esto apunta que la transformación educativa vista desde lo que implica asumir nuevos 
paradigmas y enfoques gnoseológicos, ontoepistémicos y por ende metodológicos, sustentadores de los procesos pedagógicos y didácticos” (p. 11).

Esto implica repensar el actuar de la educación desde lo docente, para Romero \& Villasmil (2019), “la reflexión sobre la formación docente a la luz de la fenoménica aleatoria del actual contexto educativo amerita de la puesta en práctica de una nueva lógica o marco de referencia" (p. 143). Es necesaria la reflexión docente como praxis profesional, lo cual posibilita involucrar aspectos relacionados al manejo de las diversas adversidades que puedan tener los estudiantes en conformidad de ayudarlos a superarlas, sobre todo cuando estas se tratan de procesos cotidianos y complejos como la lecto escritura, para lo cual se requiere que la persona tenga capacidades óptimas que le permitan desarrollar un proceso ajustado a los criterios de evaluación de la educación formal.

\section{Comprensión lectora}

En cuanto a la comprensión lectora Charmeux (1992), plantea que "leer es comprender, siempre que se lee se hace para entender sino carecería de sentido" ( $p$. 13). Un lector comprende un texto cuando puede encontrarle significado, cuando puede ponerlo en relación con lo que ya sabe y con lo que le interesa. La comprensión se vincula entonces estrechamente con la visión que cada uno tiene del mundo y de sí mismo, por lo tanto, ante un mismo texto, no se puede pretender una interpretación única y objetiva.

La comprensión lectora es importante dentro del proceso que deben desarrollar los estudiantes para poder lograr una buena expresión oral en sus relaciones en el aula y fuera de ella, el leer no es solo reproducir una serie de palabras, es comprender lo que estas quieren decir, de allí que los docentes deben tener muy en cuenta que los estudiantes deben asumir un papel protagónico en el acto de la lectura, el cual no puede ser aislado o realizado solo cuando se pretenda evaluar un determinado contenido, la lectura es el medio por el cual los estudiantes pueden llegar a tener una excelente expresión oral.

Así mismo, Charmeux (1992), manifiesta que leer es construir por sí mismo el sentido 
de un mensaje que se necesita en un proyecto dado. Leer en voz alta es comunicar oralmente a una audiencia que ha expresado su deseo, la propia lectura de un texto. Esta autora hace referencia de que el lector debe saber diferenciar entre lectura en voz alta y oralización, por cuanto manifiesta que para leer en voz alta hay que saber leer considerándola además como un actividad difícil, que exige no solo la comprensión previa del texto leído sino una toma de conciencia de dicha comprensión y un descentramiento de la misma.

De allí que la autora afirme que el leer en voz alta es una actividad interesante, rica, difícil, que debe contar con técnicas específicas para que pueda darse de la manera más óptima posible. Por lo planteado por Charmeux, la oralización consiste en leer voz alta pero de una forma mecánica; es decir, solo el lector logra la transformación de signos escritos en signos sonoros, por lo que llega a la conclusión que oralizar es la actividad escolar por excelencia, pero que no se trata ni de leer en voz baja y en voz alta, convirtiéndose así en un mecanismo carente de interés por parte del estudiante, lo que impide que se dé tanto la lectura en voz baja (interior) como en voz alta.

Esta teoría permite evidenciar como efectivamente existe una marcada diferencia entre lo que es el acto de leer propiamente dicho como tal y el acto de solo repetir mecánicamente las palabras, lo que conlleva esto último a que muchas personas piensen equivocadamente que leen cuando solo hacen oralidad o repetición mecánica de lo escrito sin ningún tipo de comprensión, lo que impide lógicamente un aprendizaje significativo en la persona, lo que a su vez conduce al fracaso académico de muchos estudiantes. Para un buen proceso de comprensión lectora Charmeux (1992, p. 49), propone los siguientes componentes, a los cuales llama los componentes del saber leer:

- Dimensión afectiva: Esta dimensión es importante y debe ser manejada por el docente para motivar al estudiante a la lectura, pero sobre todo a brindarle seguridad para que no se encierre en sí mismo y pueda brindar todo el potencial que en él existe, es ir contra la monotonía de la vida diaria, es romper con los paradigmas existenciales que pueda poseer el estudiante. 
- Dimensión cognitiva: Es importante desde el punto de vista que no puede existir comprensión lectora si la persona no tiene la capacidad para tal, por otro lado esta dimensión abarca el hecho de que el estudiante debe conceptualizar conocimientos para luego transformarlos en construcciones de ideas que le permitan tener una idea reflexiva del tema que lee, para lograr comprender a plenitud lo que está leyendo, ya que de lo contrario se quedaría en un mero acto de oralidad.

- Dimensión pragmática: el lector debe llevar a la práctica diaria la lectura con la finalidad de convertirla en hábito, de esta manera el lector logrará la soltura en el manejo de la lectura y lo que se encuentra inmersa en esta como libros, revistas, periódicos, diccionarios, entre otros. En esta dimensión el lector debe ir descubriendo el placer por leer, lo que a su vez le potenciará todo el intelecto para transformarlo en una persona que pueda responder a las diversas exigencias que la vida diaria le va fomentando.

El leer es un acto que implica ir más allá del simple hecho de repetir o reproducir unos simples signos que están escritos, es ir al significado de los mismos y trasmitirlos a los oyentes de tal manera como si éstos estuviesen realizando ellos mismos el acto de leer. De lo contrario, la expresión oral de la persona que está leyendo se realiza de una forma unidireccional, es decir, de él para él, sin lograr transmitir nada a la audiencia, otro problema que ayuda a solventar la práctica de la lectura es que ayuda a vencer el miedo que los estudiantes muchas veces padecen y no les permite leer en público por temor a convertirse en el hazme reír de los compañeros, por cuanto no poseen seguridad en sí mismos o en otros casos lo hacen de una manera cortada.

\section{CONCLUSIONES}

Las dificultades de aprendizaje durante el proceso lector son el resultado acumulado de varios factores, entre ellos la aplicación incorrecta del test de APGAR al momento del nacimiento, presencia de asfixia e hipoxia perinatal, factores ambientales, culturales y sociales a los que el escolar se ve sujeto, provocando en su trayecto lecto- 
escritor deficiencias y alteraciones que sin un plan de intervención psicopedagógico no podrá marcar un ritmo de aprendizaje adecuado.

El test de APGAR, es un test que debería ser aplicado de manera responsable y a tiempo, cuyos resultados tienen que ser conocidos por la madre o el tutor del recién nacido, para que a futuro se pueda establecer un plan de diagnóstico e intervención adaptado a las necesidades del RN.

Los niños que padecen estos trastornos o dificultades de aprendizaje, necesitan adaptaciones curriculares que los ayuden a superar este déficit, apoyados por el sistema educativo y el entorno familiar, dichas dificultades son superadas, cuando se diagnostican a tiempo.

Es necesario que la madre conozca el resultado de la valoración de APGAR del recién nacido, y que el grupo obstetra derive a centros de estimulación temprana para la ejecución de terapias que ayuden al niño a superar dificultades de aprendizaje, problemas motores, sensoriales y de adaptación, por cuanto "la precisión e inmediatez de la detección de las dificultades dependerá, en gran medida, su evolución" (Tamayo Lorenzo, 2017, p. 430), lo cual evidencia la importancia de atender a temprana edad la problemática, aunado a un buen manejo humano pedagógico en la escuela, lo cual permitirá contribuir en la integración del estudiante, así como de motivarse al aprendizaje, adquisición de hábito de lectura como medio para aprender para la vida, ejecutándose una educación inclusiva de calidad.

\section{REFERENCIAS CONSULTADAS}

1. Charmeux, E. (1992). Cómo fomentar los hábitos de lectura. Ediciones CEAC, S.A., Barcelona, España.

2. Hernández Velázquez, Norbelis, Landrove Borjas, Idalmis, \& Andrés Matos, Andrés. (2014). Desarrollo psicomotor al año de edad en niños con antecedentes de asfixia al nacer. Correo Científico Médico, 18(3), 469-478. Recuperado de http://scielo.sld.cu/scielo.php?script=sci_arttext\&pid=S1560$43812014000300008 \& \operatorname{lng}=e s \&$ lng $=e s$ 
3. lanni Gómez., L. (2018). MIRAMDA, una propuesta educativa emergente desde la investigación. Revista Arbitrada Interdisciplinaria Koinonía, 2(3), 9-30. Recuperado de http://fundacionkoinonia.com.ve/ojs/index.php/revistakoinonia/article/view/49/3 6

4. INEC (2012). 3 de cada 10 ecuatorianos no destinan tiempo a la lectura. Recuperado de https://www.ecuadorencifras.gob.ec/3-de-cada-10ecuatorianos-no-destinan-tiempo-a-la-lectura/

5. Luna, D. (2005). ¿Qué es el Score de Apgar? Recuperado de https://www.intramed.net/contenidover. asp? contenidoid=34733

6. Piaget, J. (1987) El lenguaje y el pensamiento en el niño. Paidós .Barcelona.

7. Romero, M., \& Villasmil, J. (2017). Repensar la formación docente. Hacia el encuentro de una nueva perspectiva epistémica para su abordaje y resignificación. CIENCIAMATRIA, 3(5), 133-149. Recuperado a partir de http://cienciamatriarevista.org.ve/index.php/cm/article/view/17

8. Podestá L, Loreto, Alarcón, Ana María, Muñoz, Sergio, Legüe C, Marcela, Bustos, Luis, \& Barría P, Mauricio. (2013). Psychomotor development in offspring of mothers with post partum depression. Revista médica de Chile, 141(4), 464-470. https://dx.doi.org/10.4067/S0034-98872013000400007

9. Scrich Vázquez, Aldo Jesús, Cruz Fonseca, Leticia de los Ángeles, Bembibre Mozo, Dayamí, \& Torres Céspedes, Iselkis. (2017). La dislexia, la disgrafia y la discalculia: sus consecuencias en la educación. Revista Archivo Médico de Camagüey, 21(1), 766-772. Recuperado de http://scielo.sld.cu/scielo.php?script=sci_arttext\&pid=S102502552017000100003\&lng=es\&tlng=es.

10. Tamayo Lorenzo, S. (2017). La dislexia y las dificultades en la adquisición de la lectoescritura. Recuperado de https://recyt.fecyt.es/index.php/profesorado/article/view/58074/35586 


\section{REFERENCES CONSULTED}

1. Charmeux, E. (1992). How to encourage reading habits. Ediciones CEAC, S.A., Barcelona, Spain.

2. Hernández Velázquez, Norbelis, Landrove Borjas, Idalmis, \& Andrés Matos, Andrés. (2014). Psychomotor development at one year of age in children with a history of asphyxiation at birth. Medical Scientific Mail, 18 (3), 469-478. Retrieved from http://scielo.sld.cu/scielo.php?script=sci_arttext\&pid=S1560$43812014000300008 \& \operatorname{lng}=$ en\&tIng=en

3. lanni Gómez., L. (2018). MIRAMDA, an educational proposal emerging from research. Interdisciplinary Arbitrated Review Koinonía, 2 (3), 9-30. Recovered from

http://fundacionkoinonia.com.ve/ojs/index.php/revistakoinonia/article/view/49/3 6

4. INEC (2012). 3 out of 10 Ecuadorians do not devote time to reading. Recovered from https://www.eficienterencifras.gob.ec/3-de-cada-10-ecuatorianos-nodestinan-tiempo-a-la-lectura/

5. Luna, D. (2005). What is the Apgar Score? Recovered from https://www.intramed.net/contenidover.asp?contenidoid=34733

6. Piaget, J. (1987) Language and thought in the child. Paidós .Barcelona.

7. Romero, M., \& Villasmil, J. (2017). Rethink teacher training. Towards the meeting of a new epistemic perspective for its approach and resignification. SCIENCE, $\quad 3 \quad$ (5), 133-149. Recovered from http://cienciamatriarevista.org.ve/index.php/cm/article/view/17

8. Podestá L, Loreto, Alarcón, Ana María, Muñoz, Sergio, Legüe C, Marcela, Bustos, Luis, \& Barría P, Mauricio. (2013). Psychomotor development in offspring of mothers with post partum depression. Medical Journal of Chile, 141 (4), 464-470. https://dx.doi.org/10.4067/S0034-98872013000400007

9. Scrich Vázquez, Aldo Jesús, Cruz Fonseca, Leticia de los Ángeles, Bembibre Mozo, Dayamí, \& Torres Céspedes, Iselkis. (2017). Dyslexia, dysgraphia and dyscalculia: its consequences in education. Medical Archive Magazine of $\begin{array}{lllll}\text { Camagüey, } & 21 & (1), & 766-772 . & \text { Retrieved from }\end{array}$ http://scielo.sld.cu/scielo.php?script=sci_arttext\&pid=S1025-

02552017000100003\&lng=en\&tlng=en. 


\section{CIENCIAMATRIA}

Revista Interdisciplinaria de Humanidades, Educación, Ciencia y Tecnología

Año V. Vol. V. N9. Julio - Diciembre 2019

Hecho el depósito de ley: pp201602FA4721

ISSN-L: 2542-3029; ISSN: 2610-802X

Universidad Nacional Experimental Francisco de Miranda (UNEFM). Santa Ana de Coro. Venezuela

Deysi Rosario Basantes Moscoso; Guillermo Gonzalo Gualpa Jaramillo; Guillermo Euclides Galecio Samaniego

10. Tamayo Lorenzo, S. (2017). Dyslexia and difficulties in acquiring literacy. Recovered

from https://recyt.fecyt.es/index.php/profesorado/article/view/58074/35586

(C2020 por los autores. Este artículo es de acceso abierto y distribuido según los términos y condiciones de la licencia Creative Commons Atribución-NoComercial-Compartirlgual 4.0 Internacional (CC BY-NC-SA 4.0) (https://creativecommons.org/licenses/by-nc-sa/4.0/). 